\title{
Preoperative Anemia Is Associated with Increased Mortality Following Primary Unilateral Total Joint Arthroplasty
}

\author{
Michael D. Herrick*, Brian D. Sites, Melissa M. Masaracchia, Wayne E. Moschetti \\ Departments of Anesthesiology and Orthopaedics, Dartmouth-Hitchcock Medical Center, Lebanon, NH, USA \\ Email: *Michael.D.Herrick@hitchcock.org
}

Received 10 May 2016; accepted 6 June 2016; published 9 June 2016

Copyright (C) 2016 by authors and Scientific Research Publishing Inc.

This work is licensed under the Creative Commons Attribution International License (CC BY). http://creativecommons.org/licenses/by/4.0/

(c) (i) Open Access

\begin{abstract}
Background: Total joint arthroplasty is a commonly performed procedure for end-stage osteoarthritis. Preoperative anemia is a well-characterized and potentially modifiable risk factor for morbidity and mortality in non-cardiac surgery. This retrospective cohort study identified the prevalence of anemia in our total joint arthroplasty population and investigated if there was an association with all-cause mortality. Study Design and Methods: Using an electronic medical record, we examined all patients who underwent a primary unilateral total joint arthroplasty at Dartmouth-Hitchcock Medical Center from January 1, 2011 through July 1, 2015. Preoperative anemia thresholds were defined according to the World Health Organization as $12 \mathrm{mg} / \mathrm{dl}$ for women and $13 \mathrm{mg} / \mathrm{dl}$ for men. Kaplan-Meier survival analyses were performed to examine the relationship between preoperative anemia and all-cause mortality. Cox proportional hazards were calculated for various models adjusting for potential confounders. Results: 439 of the 3247 patients $(13.5 \%)$ that underwent total joint arthroplasty met the preoperative definition for anemia. 48 patients $(1.48 \%)$ died during 6470 patient years of follow up, generating an incidence rate of 7.4 deaths per 1000 patient years. The crude hazard ratio for death in anemic patients was 3.42 (95\% CI 1.89, 6.82). In multiple models adjusting for various health related confounders, preoperative anemia was associated with a roughly two-fold increase risk of death compared to nonanemic patients. Conclusion: Preoperative anemia is prevalent in our population and it is associated with increased postoperative mortality in total joint arthroplasty patients, even when adjustments are made for significant co-morbidities.
\end{abstract}

\section{Keywords}

Anesthesia, Total Joint Arthroplasty, Anemia

\footnotetext{
${ }^{*}$ Corresponding author.
}

How to cite this paper: Herrick, M.D., Sites, B.D., Masaracchia, M.M. and Moschetti, W.E. (2016) Preoperative Anemia Is Associated with Increased Mortality Following Primary Unilateral Total Joint Arthroplasty. Open Journal of Anesthesiology, 6, 91-96. http://dx.doi.org/10.4236/ojanes.2016.66015 


\section{Introduction}

Total joint arthroplasty (TJA) is one of the most commonly performed procedures in the world. According to the Centers for Disease Control, there were over 1 million total knee and hip procedures performed in the United States in 2010 [1]. As of 2010, there were an estimated 7.2 million Americans living with a total knee or hip replacement, and, by 2020, two million Americans will undergo a hip or knee replacement each year [2]. Although total joint replacement is a highly effective treatment for end-stage osteoarthritis, patients can experience significant perioperative morbidity and mortality. According to one large retrospective analysis of 15,321 patients, between $1.6 \%$ and $2.1 \%$ of patients will experience a major systemic complication following knee arthroplasty (TKA) [3]. Although rare, 30 -day mortality is estimated to be $0.3 \%$ following total hip arthroplasty (THA) [4] and $0.18 \%$ following total knee replacement [3].

A robust body of literature has identified multiple risk factors associated with complications following TJA. Age and diabetes are independent risks factors for mortality following TKA [3]. Additionally, an increased risk of 90-day mortality following THR is associated with patients who have one of the following comorbid conditions: congestive heart failure, metastatic cancer, psychosis, renal disease, dementia, hemiplegia, cerebrovascular disease or chronic pulmonary disease [5]. Pre-existing conditions that have been associated with postoperative joint infections include rheumatologic disease, obesity, coagulopathy and preoperative anemia [5]. Of all the complications associated with TJR, death is the least studied. This is partially a function of the rare nature of the event, generating suboptimal power to understand the relationship to various exposures. Risk factors combined, with expert opinion, have been utilized to create multiple clinical pathways aimed at decreasing morbidity [6][8]. Decreasing mortality after TJA is an important outcome because over 1000 Americans will die each year within 30 days following a TJA [2]-[4]. Strategies to reduce this mortality are thus warranted.

In the past several years, preoperative anemia has been identified as a risk factor for morbidity and mortality in non-cardiac surgery [9]. Preoperative anemia is an attractive risk factor to understand because it can potentially be modified prior to elective surgery. In the orthopedic population, preoperative anemia has been linked to higher rates of transfusion, longer lengths of stay, and an increased risk of wound infection [10] [11]. However, long-term survival outcome data in the setting of preoperative anemia is limited, likely by both the rarity of the event and emphasis on 30 or 60-day survival metrics. As part of an exploratory quality improvement initiative, we conducted a retrospective cohort study with the following goals: 1 ) to identify the prevalence of preoperative anemia in our total joint population; 2) to determine whether preoperative anemia is associated with increased mortality in these patients.

\section{Materials and Methods}

Following approval and a consent waiver from the Dartmouth College Committee for the Protection of Human Subjects, (63 South Main Street, Hanover, NH 03755 USA; Study \#00028930 approved on September 16, 2015) we examined 3247 patients undergoing primary unilateral TJA at a single institution from January 1, 2011 through July 1, 2015. Dartmouth-Hitchcock Medical Center is a tertiary care academic medical center located in Lebanon, New Hampshire, USA. Revision surgeries and bilateral procedures were excluded in the analysis. The data set generated from our electronic medical record (Epic Systems Corp.) included standard demographics, medical comorbidities, transfusion events, mortality, and laboratory data. The hemoglobin closest to the time prior to surgery was used for this study. Patients that did not have a hemoglobin value within six months prior to surgery were excluded. The decision to transfuse the patient was made by the primary anesthesia team.

\section{Statistical Analysis}

Stata Version 12 (StataCorp, College Station, TX, USA) was used for the statistical analysis. For the descriptive statistics, categorical variables are summarized as proportions and continuous variables are reported as means and standard deviations. The prevalence of preoperative anemia was measured, and defined as a hemoglobin concentration threshold of the World Health Organization's gender-based definition of $12.0 \mathrm{~g} / \mathrm{dl}$ in women and $13.0 \mathrm{~g} / \mathrm{dl}$ in men [12]. Univariate analysis, using chi-square statistic for categorical variables, and the test for continuous variables, was performed to characterize the relationship of anemic status to patient characteristics (Table 1).

Kaplan-Meier curves were used to describe survival by preoperative anemia status (anemic versus not anem- 
Table 1. Characteristics of patients having total joint arthroplastyby anemia status (2011-2015).

\begin{tabular}{|c|c|c|c|}
\hline \multicolumn{4}{|c|}{ Mean (SD) or proportion } \\
\hline Characteristic & $\begin{array}{c}\text { Anemic }^{\infty} \\
\mathrm{n}=439\end{array}$ & $\begin{array}{c}\text { Not Anemic }^{\infty} \\
n=2801\end{array}$ & $\begin{array}{c}\text { All } \\
\mathrm{n}=3247\end{array}$ \\
\hline Age (years) & $62.5(10.9)^{*}$ & $63.4(10.7)$ & $63.9(10.9)$ \\
\hline Sex (male) & 0.435 & 0.455 & 0.452 \\
\hline BMI & $30.9(6.9)$ & $31.1(7.5)$ & $31.05(7.7)$ \\
\hline Race (white) & 0.996 & 0.989 & 0.988 \\
\hline ASA III and higher" & $0.585^{*}$ & 0.362 & 0.392 \\
\hline Diabetes & $0.196^{*}$ & 0.113 & 0.125 \\
\hline Cancer (any) & 0.088 & 0.074 & 0.047 \\
\hline Coronary artery disease & 0.164 & 0.134 & 0.138 \\
\hline Tobacco smoking & 0.041 & 0.036 & 0.036 \\
\hline COPD & $0.071^{*}$ & 0.044 & 0.047 \\
\hline Renal insufficiency & $0.102^{*}$ & 0.023 & 0.033 \\
\hline Congestive heart failure & $0.062^{*}$ & 0.011 & 0.018 \\
\hline Transfusion $\odot$ & $0.303^{*}$ & 0.061 & 0.938 \\
\hline \multicolumn{4}{|l|}{ Surgery type } \\
\hline Anterior total hip & $0.237^{*}$ & 0.329 & 0.317 \\
\hline Posterior total hip & $0.243^{*}$ & 0.317 & 0.252 \\
\hline Total knee & 0.447 & 0.427 & 0.430 \\
\hline Mortality (any day) & $0.387^{*}$ & 0.111 & 0.148 \\
\hline
\end{tabular}

\footnotetext{
"Based on American Society of Anesthesiologists Health Classification; ${ }^{\infty}$ Anemia based on World Health Organization Classification of $\leq 12 \mathrm{mg} / \mathrm{dl}$ for women and $\leq 13 \mathrm{mg} / \mathrm{dl}$ for men; ${ }^{*}$ p value $<0.05$ comparing anemic to not anemic patients; $\odot$ Defined as any packed red blood cell transfusion during hospital admission.
}

ic). The log-rank test and Cox Proportional Hazards modeling were used for univariate and multivariate tests of statistical significance, respectively. Our a priori assumption was that there was co-linearity between anemic status and the occurrence of a postoperative transfusion. We thus plotted a separate survival curve for anemic patients by transfusion status. Transfusion was defined as administration of packed red blood cells at any time during the index hospital admission.

For all analyses, we set the p-value for statistical significance to 0.05 (2-sided).

\section{Results}

We identified 3247 patients that underwent primary TJA between January 1, 2011, and July 1, 2015. 530 patients had more than one joint replacement during this period. 48 patients (1.48\%) died during 6470 patient years of follow up, generating an incidence rate of 7.4 deaths per 1000 patient years. Overall mortality as a function of age and time period is demonstrated in Table 2.

439 of the 3247 patients met the WHO criteria for anemia, generating a prevalence of $13.5 \%$. A preoperative hemoglobin was unable to be obtained for seven patients. Anemic patients were slightly younger than the non-anemic patients (62.5 yrs. vs. 63.4 yrs.), and had higher rates of diabetes, COPD, renal insufficiency and congestive heart failure (Table 1). Patients with preoperative anemia were approximately five times more likely to receive a transfusion ( $30 \%$ vs. $6 \%$ ). 
The Kaplan-Meier curve (Figure 1) demonstrates an increased risk of mortality associated with patients who were anemic prior to their surgery ( $p$ value $<0.001$ ). The crude hazard ratio (HR) for preoperative anemia was 3.42 (95\% CI 1.89 - 6.2). To adjust for the American Society of Anesthesiologists (ASA) health score, as well as for several pre-operative comorbid conditions, we performed several regression analyses (Table 3). The HR decreased to 2.14 (95\% CI 1.13, 4.07) when adjusted for ASA score and to 2.45 (95\% CI 1.35, 4.46) when adjusted for a combination of diabetes mellitus, coronary artery disease, and COPD (Table 3).

The influence of transfusion on all-cause mortality was also explored. Patients having at least one packed red blood cell transfusion at anytime during their hospital stay were approximately two times more likely to die within the follow up period(HR 2.19; 95\%CI 1.12, 4.27). Figure 2 demonstrates that anemic patients who received a transfusion were 4.3 times more likely to die compared to anemic patients who did not get a transfusion (p value < 0.001HR 4.3; 95\% CI: 1.5, 12.2). Additionally, non-anemic patients who received a transfusion were approximately 3 times more likely to die compared to non-anemic patients who did not get a transfusion (p value $=0.005,95 \%$ CI: $1.43,7.76$ ).

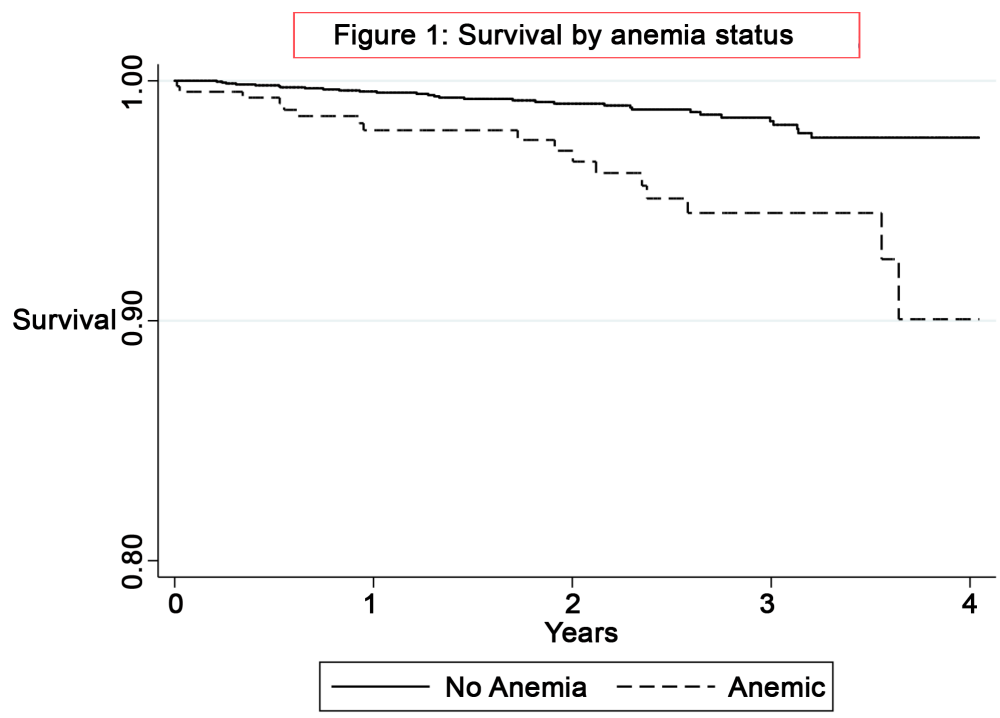

Figure 1. Kaplan-Meier survival curves by preoperative anemia status.

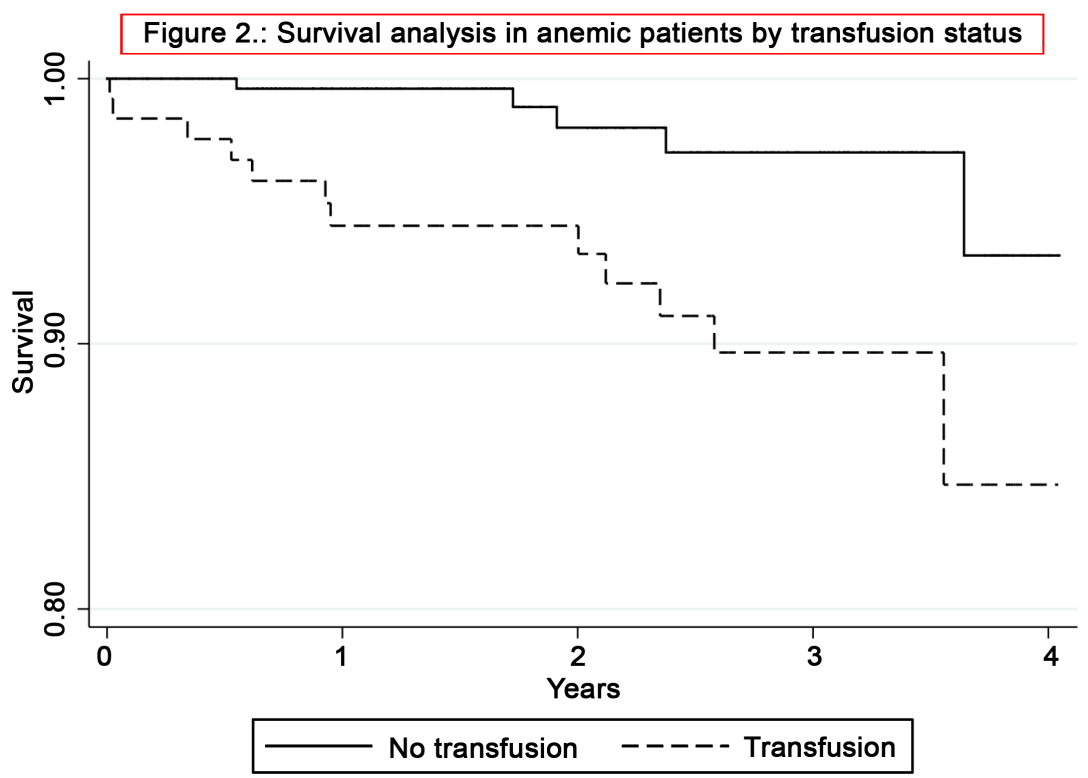

Figure 2. Kaplan-Meier survival curves for preoperative anemic patients by transfusion status. 
Table 2. Mortality by age strata (April, 2011-March, 2015) ${ }^{€}$ in total joint arthroplasty patients.

\begin{tabular}{cccccc}
\hline & \multicolumn{5}{c}{$\mathbf{N},(\%)$} \\
\hline Age Category $^{\boldsymbol{x}}(\mathbf{N})$ & $\mathbf{3 0 - \text { -day }}$ & $\mathbf{6 0 - d a y}$ & $\mathbf{9 0 - d a y}$ & One-year & Any-day \\
\hline$<55(601)$ & $0,(0.00)$ & $0,(0.00)$ & $0,(0.00)$ & $2,(0.33)$ & $3,(0.50)$ \\
$55-65(1294)$ & $0,(0.00)$ & $0,(0.00)$ & $1,(0.08)$ & $3,(0.25)$ & $7,(0.58)$ \\
$>65(1442)$ & $2,(0.14)$ & $2,(0.14)$ & $3,(0.12)$ & $12,(0.83)$ & $38,(2.64)^{*}$ \\
Any age & $2,(0.06)$ & $2,(0.06)$ & $4,(0.12)$ & $17(0.52)$ & $48,(1.48)$ \\
\hline
\end{tabular}

${ }^{\epsilon}$ Includes both unilateral primary total knee and total hip arthroplasty; ${ }^{\mathrm{k}}$ Years; ${ }^{*} \mathrm{p}$ value $<0.05$ when compared to $<55$ years old.

Table 3. Regression analysis of anemia model predicting mortality.

\begin{tabular}{cccc}
\hline & Hazard Ratio & 95\% CI & \multicolumn{1}{c}{ P value } \\
\hline Anemia (unadjusted) & 3.42 & $1.89,6.2$ & 0.000 \\
Model A & 2.14 & $1.13,4.07$ & 0.019 \\
Model B $\odot$ & 2.45 & $1.35,4.46$ & 0.003 \\
Model C & 2.97 & $1.64,5.40$ & 0.000 \\
Model D & 2.85 & $1.52,5.32$ & 0.001 \\
Model C & 2.19 & $1.12,4.27$ & 0.021 \\
\hline
\end{tabular}

${ }^{\infty}$ Adjusted for age, body mass index; $\odot$ Adjusted for advanced ASA status ( $\geq 3$ ); ${ }^{\sharp}$ Adjusted for diabetes mellitus, coronary artery disease, and COPD; ${ }^{€}$ Adjusted for diabetes mellitus, congestive heart failure, and renal insufficiency; ${ }^{\mu}$ Adjusted for receiving a transfusion at any time during hospital postoperative course.

\section{Discussion}

We identified a high prevalence of preoperative anemia in patients undergoing TJA at our medical center. Preoperative anemia was associated with an increased risk of death as determined by a formal time to event analysis over the 4-year study period. The risk of death appeared independent of the presence of co-morbid medical conditions. We further identified that both preoperative anemic and non-anemic patients who received a blood transfusion were at an additional increased risk of death.

To our knowledge, this is the first observational data set including all patients undergoing TJA that has demonstrated an increased risk of mortality associated with preoperative anemia. Previous TJA outcomes research has focused on other metrics, partially because many large publicly available datasets such as the $5 \%$ Medicare sample do not track hemoglobin levels [3] [5] [13].

Our data is observational in nature and a causal relationship between anemia and death cannot be proven. Additionally, the observed relationship between anemia and death may be confounded by unmeasured variables. Given that our total death event number is 48 , we are limited in our ability to model and control for additional confounders. Moreover, even if we assume a cause and effect relationship between anemia and death, it is unclear if correcting preoperative anemia will improve on mortality.

Despite these limitations, these data allow us to better educate patients and the care team regarding risks associated with TJA. Given that TJA is an elective procedure, our health system is collaboratively creating a care pathway based on an algorithm from The Network for Advancement of Transfusion Alternatives [14]. This process will allow us to further investigate patients who have been identified as anemic. Patients found to have reversible causes of anemia, such as iron deficiency, can be treated prior to the surgical intervention. We are also exploring the use of erythropoietin in certain anemic patient populations. Our future work will be to track mortality and the prevalence of anemia to assess the impact of our various interventions.

\section{Disclaimer}

This work was supported by the Dartmouth-Hitchcock Department of Anesthesiology D. David Glass Scholarship. 


\section{References}

[1] Centers for Disease Control and Prevention: Fast Stats. http://www.cdc.gov/nchs/fastats/inpatient-surgery.htm

[2] Kremers, H., Larson, D., Crowson, C., Kremers, W., Washington, R., Steiner, C., Jiranek, W. and Berry, D. (2015) Prevalence of Total Hip and Knee Replacement in the United States. The Journal of Bone \& Joint Surgery, 97, 13861397. http://dx.doi.org/10.2106/JBJS.N.01141

[3] Belmont Jr., P., Goodman, G., Waterman, B., Bader, J. and Schoenfeld, A. (2014) Thirty-Day Postoperative Complications and Mortality Following Total Knee Arthroplasty. The Journal of Bone \& Joint Surgery, 96, 20-26. http://dx.doi.org/10.2106/JBJS.M.00018

[4] Berstock, J., Beswick, A., Lenguerrand, E., Whitehouse, M. and Blom, A. (2014) Mortality after Total Hip Replacement Surgery: A Systematic Review. Bone \& Joint Research, 3, 175-182. http://dx.doi.org/10.1302/2046-3758.36.2000239

[5] Bozic, K., Lau, E., Kurtz, S., Ong, K., Rubash, H., Vail, T. and Berry, D. (2012) Patient-Related Risk Factors for Periprosthetic Joint Infection and Postoperative Mortality Following Total Hip Arthroplasty in Medicare Patients. The Journal of Bone \& Joint Surgery, 94, 794-800. http://dx.doi.org/10.2106/JBJS.K.00072

[6] Gooch, K., Marshall, D., Faris, P., Khong, H., Wasylak, T., Pearce, T., Johnston, D., Arnett, G., Hibbert, J., Beaupre, L., Zernicke, R. and Frank, C. (2012) Comparative Effectiveness of Alternative Clinical Pathways for Primary Hip and Knee Joint Replacement Patients: A Pragmatic Randomized, Controlled Trial. Osteoarthritis Cartilage, 20, 1086-1094. http://dx.doi.org/10.1016/j.joca.2012.06.017

[7] Van Citters, A., Fahlman, C., Goldmann, D., Lieberman, J., Koenig, K., DiGioia, A., O’Donnell, B., Martin, J., Federico, F., Bankowitz, R., Nelson, E. and Bozic, K. (2014) Developing a Pathway for High-Value, Patient-Centered Total Joint Arthroplasty. Clinical Orthopaedics and Related Research, 472, 1619-1635. http://dx.doi.org/10.1007/s11999-013-3398-4

[8] Hass, D., Helmers, R., Rucci, M., Brady, M. and Kaplan, R. (2015) The Mayo Clinic Model for Running a Value-Improvement Program. https://hbr.org/2015/10/the-mayo-clinic-model-for-running-a-value-improvement-program

[9] Beattie, W., Karkouti, K., Wijeysundera, D. and Tait, G. (2009) Risk Associated with Preoperative Anemia in Noncardiac Surgery. Anesthesiology, 110, 574-581. http://dx.doi.org/10.1097/ALN.0b013e31819878d3

[10] Greenky, M., Gandhi, K., Pulido, L., Restrepo, C. and Parvizi, J. (2012) Preoperative Anemia in Total Joint Arthroplasty: Is It Associated with Periprosthetic Joint Infection? Clinical Orthopaedics and Related Research, 470, 26952701. http://dx.doi.org/10.1007/s11999-012-2435-Z

[11] Jans, O., Jorgensen, C., Kehlet, H. and Johansson, P. (2014) Role of Preoperative Anemia for Risk of Transfusion and Postoperative Morbidity in Fast-Track Hip and Knee Arthroplasty. Transfusion, 54, 717-726. http://dx.doi.org/10.1111/trf.12332

[12] WHO (2011) Haemoglobin Concentrations for the Diagnosis of Anaemia and Assessment of Severity. Vitamin and Mineral Nutrition Information System, World Health Organization (WHO/NMH/NHD/MNM/11.1), Geneva. http://www.who.int/vmnis/indicators/haemoglobin.pdf

[13] Yu, S., Garvin, K., Healy, W., Pellegrini Jr., V. and Iorio, R. (2015) Preventing Hospital Readmissions and Limiting the Complications Associated with Total Joint Arthroplasty. Journal of the American Academy of Orthopaedic Surgeons, 23, e60-e71. http://dx.doi.org/10.5435/JAAOS-D-15-00044

[14] Goodnough, L., Maniatis, A., Earnshaw, P., Benoni, G., Beris, P., Bisbe, E., Fergusson, D., Gombotz, H., Habler, O., Monk, T., Ozier, Y., Slappendel, R. and Szpalski, M. (2011) Detection, Evaluation, and Management of Preoperative Anaemia in the Elective Orthopaedic Surgical Patient: NATA Guidelines. British Journal of Anaesthesia, 106, 13-22. http://dx.doi.org/10.1093/bja/aeq361 